\title{
SEMINORMS AND THE EGOROFF PROPERTY IN RIESZ SPACES
}

BY

JOHN A. R. HOLBROOK

1. Introduction. The following discussion is set in the theory of Riesz spaces, i.e. real linear spaces with compatible lattice structures. An order structure being available, it is natural to require seminorms on a Riesz space $L$ to be monotone; specifically we say that $\rho$ is a monotone seminorm on $L$ if, as usual, $\rho$ is a positive homogeneous and sublinear function on $L$, with range in $[0,+\infty]$ (note that $+\infty$ is admitted as a value), and, in addition, $|g| \leqq|f| \Rightarrow \rho(g) \leqq \rho(f)$. If $\rho$ is "continuous" to the extent that $0 \leqq f_{n} \uparrow f \Rightarrow \rho\left(f_{n}\right) \uparrow \rho(f)$, we say (following the terminology of Luxemburg and Zaanen $[6, \S 5])$ that $\rho$ is $\sigma$-Fatou. It is easily shown that for each monotone seminorm $\rho$ there is a corresponding $\rho_{M}$ which is maximal among the $\sigma$-Fatou monotone seminorms dominated by $\rho$. The main question treated here is: in what cases can $\rho_{M}$ be obtained via the natural construction

$$
\rho_{L}(u)=\inf \left\{\lim _{n} \rho\left(u_{n}\right): 0 \leqq u_{n} \uparrow u\right\},
$$

i.e. when do we have $\rho_{L}=\rho_{M}$ ? A practical answer is given in terms of the almostEgoroff property, a condition on the order structure of the space $L$ which is a variant of the Egoroff condition introduced by Nakano and by Luxemburg for other purposes (see \$2). In fact, we have the following theorem, as a consequence of results proved in $\S 5$.

THEOREM 1.1. Let $L$ be a Riesz space. Then $\rho_{M}=\rho_{L}$ for every monotone seminorm $\rho$ on $L$ if, and only if, $L$ is almost-Egoroff.

The more significant part of this theorem is the necessity of the almost-Egoroff condition. This shows, for example, that even such a highly regarded space as $C[0,1]$ possesses a monotone seminorm $\rho$ such that $\rho_{M} \neq \rho_{L}$ (in the case of $C[0,1]$ we may also assume that $\rho$ is a norm and finite-valued everywhere). Part of the interest of these results stems from the observation of Lorentz (see [6, §7]) concerning Riesz spaces $L$ which belong to the important subclass of "Banach function spaces," i.e. spaces consisting of the real measurable functions over a given measure space (with identification of functions equal a.e.). In this case, Lorentz showed that, for each monotone seminorm $\rho$ on $L, \rho_{M}$ is just the corresponding "Lorentz seminorm" $\rho_{L}$, provided the underlying measure space is $\sigma$-finite. For Banach function spaces, Theorem 1.1 yields a weaker condition on the measure space which is exactly equivalent to the identity of $\rho_{M}$ and $\rho_{L}$ for all $\rho$ (see §3).

Received by the editors April 6, 1966 and, in revised form, February 20, 1967. 
These results form part of the author's doctoral thesis, written under the direction of Professor W. A. J. Luxemburg at the California Institute of Technology. This research was supported in part by the Ford Foundation.

2. Egoroff conditions. We shall be concerned, for the most part, with abstract Riesz spaces, for the general properties of which we refer the reader to Bourbaki [2, Chapter II], to Nakano [10], or to Luxemburg and Zaanen [6, Note $\mathrm{V}_{\mathbf{i}}^{-}$and following]. The basic Egoroff condition is a formulation, in the Riesz space setting, of the property which lies behind Egoroff's classical result (see [3]) concerning spaces of measurable functions.

It is convenient to introduce first a special notational device. If $A$ is any system with a partial ordering " $\leqq$ ", and $\left\{a_{n, k}\right\}$ is a double sequence of elements in $A$, we write $a \ll\left\{a_{n, k}\right\}$, where $a \in A$, to mean that $(\forall n)(\exists k(n))$ such that $a \leqq a_{n, k(n)}$.

Consider first a Boolean algebra $\mathscr{B}$. An element $b \in \mathscr{B}$ is said to have the Egoroff property if

$$
\left[(\forall n) b_{n, k} \uparrow_{k} b\right] \Rightarrow\left[\left(\exists b_{m}\right): b_{m} \uparrow_{m} b \text { and }(\forall m) b_{m} \ll\left\{b_{n, k}\right\}\right] .
$$

The Boolean algebra $\mathscr{B}$ itself is said to be Egoroff if every one of its elements has the Egoroff property.

In the case of a Riesz space $L$, we say that an element $u \in L^{+}$has the Egoroff property if

$$
\left[(\forall n) 0 \leqq u_{n, k} \uparrow_{k} u\right] \Rightarrow\left[\left(\exists u_{m} \geqq 0\right): u_{m} \uparrow_{m} u \text { and }(\forall m) u_{m} \ll\left\{u_{n, k}\right\}\right] .
$$

We say that the space $L$ is Egoroff if every element in $L^{+}$has the Egoroff property.

If $\mathscr{B}$ is a Boolean algebra, $b \in \mathscr{B}$ is said to have the weak Egoroff property if

$$
\left[(\forall n) b_{n, k} \uparrow_{k} b\right] \Rightarrow\left[b=0 \text { or }(\exists a \neq 0): a \ll\left\{b_{n, k}\right\}\right] .
$$

Following the pattern above, we have the definitions of weak Egoroff Boolean algebras, elements having the weak Egoroff property in a Riesz space, and weak Egoroff Riesz spaces.

It appears that Nakano was the first to isolate the Egoroff property for Riesz spaces; with certain reservations, the Egoroff property corresponds to his notion of "total continuity" (see [10, §14]). Luxemburg introduced Egoroff and weak Egoroff Boolean algebras in [8], using the present definitions. The properties also occur in the present form in Luxemburg and Zaanen [6] (see, for example, Definition 20.5).

We wish to introduce here a third Egoroff-type property, which will play an important rôle in the sequel. If $L$ is a Riesz space, we say an element $u \in L^{+}$has the almost-Egoroff property if

$$
\begin{aligned}
{\left[(\forall n) 0 \leqq u_{n, k} \uparrow_{k} u\right] } \\
\quad \Rightarrow\left[(\forall \varepsilon \text { with } 0<\varepsilon \leqq 1)\left(\exists u_{m}^{\varepsilon} \geqq 0\right): u_{m}^{\varepsilon} \uparrow_{m}(1-\varepsilon) u \text { and }(\forall m) u_{m}^{\varepsilon} \ll\left\{u_{n, k}\right\}\right] .
\end{aligned}
$$


Of course, the space $L$ is called almost-Egoroff if every element in $L^{+}$has the almostEgoroff property. For Boolean algebras, there is no useful analogue of the almostEgoroff property.

We make here the simple observation that the Egoroff property is an "ideal property".

THEOREM 2.1. (a) If $u \geqq 0$ is an element in the Riesz space $L$ and $u$ has the Egoroff property, then the ideal generated by $u$, i.e., $\{f: f \in L$ and $(\exists M):|f| \leqq M u\}$ is Egoroff. (b) If an element $b$ of the Boolean algebra $\mathscr{B}$ has the Egoroff property, then every element a such that $a \leqq b$ has also that property.

Proof. (a) We need only show that if $0 \leqq v \leqq u$, then $v$ has the Egoroff property. But if $0 \leqq v_{n, k} \uparrow_{k} v$, then $\left(v_{n, k}+(u-v)\right) \uparrow_{k} u$, and there exist $u_{m}$ such that $0 \leqq u_{m} \uparrow_{m} u$ and $u_{m} \ll\left\{\left(v_{n, k}+(u-v)\right)\right\}$; it follows that $0 \leqq\left(u_{m}-(u-v)\right)^{+} \ll\left\{v_{n, k}\right\}$ and

$$
\left(u_{m}-(u-v)\right)^{+} \uparrow_{m}(u-(u-v))^{+}=v .
$$

(b) If $a_{n, k} \uparrow_{k} a$, then $a_{n, k} \vee\left(b \wedge a^{\prime}\right) \uparrow_{k} a \vee\left(b \wedge a^{\prime}\right)=b$. Thus there exist $b_{m}$ such that $b_{m} \uparrow_{m} b$ and $b_{m} \ll\left\{\left(a_{n, k} \vee\left(b \wedge a^{\prime}\right)\right)\right\}$, so that $\left(b_{m} \wedge a\right) \uparrow_{m} a$ and

$$
\left(b_{m} \wedge a\right) \ll\left\{\left(\left(a_{n, k} \vee\left(b \wedge a^{\prime}\right)\right) \wedge a\right)\right\}=\left\{a_{n, k}\right\} .
$$

3. Banach function spaces; Egoroff's theorem. We wish to discuss here the significance of which we have called the Egoroff property for the classical case of Riesz spaces of real measurable functions. We shall also show how the Egoroff property is involved in the theorem of Egoroff, although it is not our intention to formulate generalizations of that theorem (cf. [10, Theorem 14.2]).

Given a set $X$, a $\sigma$-complete Boolean algebra $\mathscr{T}$ of subsets of $X$ which is a subalgebra of the power set $p(X)$, and a countably additive measure $\mu$ on $\mathscr{T}$, valued in $[0,+\infty]$, let $L$ denote the space of all real-valued functions on $X$, measurable with respect to $\mathscr{T}$ and finite-valued almost everywhere (a.e.), with identification of almost everywhere equal functions. With the introduction of the usual linear operations and partial ordering, $L$ becomes a Riesz space. Such a space is known as a "Banach function space" (more properly, for each monotone seminorm $\rho$ on $L$ such that $L$ is complete with respect to $\rho$ we have the Banach function space $L_{\rho}=\{f: f \in L$ and $\left.\rho(f)<\infty\}\right)$. The assumption that the measure space $(X, \mathscr{T}, \mu)$ is $\sigma$-finite is often included in the definition, but we do not wish to do this. Let $\mathscr{B}$ denote the measure algebra associated with $(X, \mathscr{T}, \mu)$, i.e., $\mathscr{B}$ is the Boolean algebra composed of equivalence classes of elements from $\mathscr{T}$, where $A, B \in \mathscr{T}$ are identified whenever the symmetric difference $A \triangle B$ has measure zero.

We shall say the Egoroff theorem holds for $(X, \mathscr{T}, \mu)$ if, whenever $F_{k}$ are measurable functions and $F_{k} \rightarrow F$ a.e., there exist $X_{m} \in \mathscr{T}$ such that $X_{m} \uparrow, \mu\left(X-\bigcup_{1}^{\infty} X_{m}\right)$ $=0$, and $F_{k} \rightarrow F$ uniformly on each $X_{m}$. This is a general form of the well-known result of Egoroff (cf. [3]) concerning a finite real interval and Lebesgue measure. 
THEOREM 3.1. If $L$ and $\mathscr{B}$ are the Riesz space and Boolean algebra associated with a measure space $(X, \mathscr{T}, \mu)$, using the notation developed above, then the following are equivalent: (a) L is Egoroff; (b) $\mathscr{B}$ is Egoroff; (c) the Egoroff theorem holds for $(X, \mathscr{T}, \mu)$

Proof. (a) $\Rightarrow$ (b): Recalling Theorem 2.1(b), we need only consider the situation where $b_{n, k} \uparrow_{k} 1$ (for each $n$ ) in $\mathscr{B}$. If we now choose $X_{n, k} \in \mathscr{T}$ such that $X_{n, k} \in b_{n, k}$ and let $u_{n, k}$ be the element of $L$ containing $X_{n, k}$ (now thought of as a measurable function; in general we use the same symbol for a set and its characteristic function), we have $0 \leqq u_{n, k} \uparrow_{k} u$ (for each $n$ ), where $u$ is the element of $L$ containing the unit function on $X$. Assuming the Egoroff property on $L$, we have $0 \leqq u_{m} \uparrow_{m} u$ such that $(\forall m) u_{m} \ll\left\{u_{n, k}\right\}$. Choosing measurable functions $F_{m} \in u_{m}$, we must have $F_{m}(x) \uparrow 1$ a.e. so that, if we let $X_{m}=\left\{x: F_{m}(x)>0\right\}$, and let $b_{m}$ be that element of $\mathscr{B}$ which contains $X_{m}$, we certainly have $b_{m} \uparrow 1$. Moreover, if we fix $m$ for a moment, for each $n$ there exists $k(n)$ such that $u_{m} \leqq u_{n, k(n)}$; hence $F_{m} \leqq X_{n, k(n)}$ a.e. so that $\mu\left(X_{m}-X_{n, k(n)}\right)=0$. It follows that $b_{m} \leqq b_{n, k(n)}$, and hence $(\forall m) b_{m} \ll\left\{b_{n, k}\right\}$.

$(b) \Rightarrow(c)$ : Given measurable functions $F_{k}$ on $X$, such that $F_{k} \rightarrow F$ a.e., set

$$
X_{n, k}=\left\{x:\left(\forall k^{\prime}\right) k^{\prime} \geqq k \Rightarrow\left|F_{k^{\prime}}(x)-F(x)\right|<1 / n\right\} .
$$

Clearly, for each $n, X_{n, k} \uparrow_{k}$ and $\mu\left(X-\bigcup_{k=1}^{\infty} X_{n, k}\right)=0$. Thus, if $b_{n, k}$ is the element of $\mathscr{B}$ such that $X_{n, k} \in b_{n, k}$, we have $b_{n, k} \uparrow_{k} 1$, for each $n$. Under the assumption that $\mathscr{B}$ is Egoroff, we have $b_{m} \uparrow_{m} 1$ with $b_{m} \ll\left\{b_{n, k}\right\}$, for each $m$. Let $k(m, n)$ be such that $k(m, n) \uparrow_{m}$ for each fixed $n$ and $b_{m} \leqq b_{n, k(m, n)}$. If we choose $Y_{m} \in b_{m}$, we must have $\mu\left(X-\bigcup_{1}^{\infty} Y_{m}\right)=0$ and, for each $m, \mu\left(Y_{m}-X_{m}\right)=0$, where $X_{m}=\bigcap_{n=1}^{\infty} X_{n, k(m, n)}$. Thus we have $\mu\left(X-\bigcup_{1}^{\infty} X_{m}\right)=0$, and $X_{m} \uparrow$ because of the choice of $k(m, n)$. Finally, $F_{k} \rightarrow F$ uniformly on each $X_{m}$, since $k \geqq k(m, n)$ and $x \in X_{m}$ imply that $\left|F_{k}(x)-F(x)\right|<1 / n$.

(c) $\Rightarrow\left(\right.$ a): Given $(\forall n) 0 \leqq u_{n, k} \uparrow_{k} u$ in $L$, we can choose measurable functions $F_{n, k} \in u_{n, k}, F \in u$ such that $(\forall x \in X)(\forall n) 0 \leqq F_{n, k}(x) \uparrow_{k} F(x)$. Let

$$
G_{n, k}=F_{1, k} \wedge F_{2, k} \wedge \cdots \wedge F_{n, k},
$$

and let $G_{k}(x)=\inf \left\{1 / n: G_{n, k}(x) \geqq F(x)-1 / n\right\}$. Now, for each $x, G_{k}(x) \rightarrow 0$, since for each $n$ there is $k(n)$ such that if $k \geqq k(n)$ then $G_{n, k}(x) \geqq F(x)-1 / n$ and hence $G_{k}(x) \leqq 1 / n$. Since the Egoroff theorem is assumed to hold, we have $X_{m} \in \mathscr{T}$ such that $X_{m} \uparrow, \mu\left(X-\bigcup_{1}^{\infty} X_{m}\right)=0$, and $G_{k} \rightarrow 0$ uniformly on each $X_{m}$. Let $F_{m}=$ $\left[(F-1 / m) X_{m}\right]^{+}$; clearly $F_{m}(x) \uparrow F(x)$ a.e. Given $m$, we have some $k(m)$ such that, for $x \in X_{m}, G_{k(m)}(x)<1 / m$ so that $(\exists n>m): G_{n, k(m)}(x) \geqq F(x)-1 / n$ and, since the functions $G_{n, k}$ decrease in $n, G_{m, k(m)}(x) \geqq F(x)-1 / m$. Hence $F_{m} \leqq G_{m, k(m)}$ and it follows that $F_{m} \ll\left\{F_{n, k}\right\}$, for if $n<m$ then $F_{m} \leqq G_{m, k(m)} \leqq G_{n, k(m)} \leqq F_{n, k(m)}$, while if $n \geqq m$ then $F_{m} \leqq F_{n} \leqq G_{n, k(n)} \leqq F_{n, k(n)}$. Finally, if we let $u_{m}$ be the element of $L$ containing $F_{m}$, we have $u_{m} \uparrow u$ and $(\forall m) u_{m} \ll\left\{u_{n, k}\right\}$. Q.E.D. 
THEOREM 3.2. If the measure space $(X, \mathscr{T}, \mu)$ is $\sigma$-finite, i.e. if there exist $X_{m} \in \mathscr{T}$ such that $X_{m} \uparrow X$ and $(\forall m) \mu\left(X_{m}\right)<\infty$, then $\mathscr{B}$ is Egoroff.

Proof. In the obvious way, $\mu$ induces on the $\sigma$-complete Boolean algebra $\mathscr{B}$ a measure, which we again denote by $\mu$; furthermore, there exists a sequence $a_{m} \uparrow 1$ such that $\mu\left(a_{m}\right)<\infty$ for each $m$. Suppose that $(\forall n) b_{n, k} \uparrow_{k} 1$. Clearly, for each $m, n,\left(a_{m} \wedge b_{n, k}^{\prime}\right) \downarrow_{k} 0$ and, as $\mu\left(a_{m} \wedge b_{n, 1}^{\prime}\right) \leqq \mu\left(a_{m}\right)<\infty$, we can choose $k(m, n)$ such that $\mu\left(a_{m} \wedge b_{n, k(m, n)}^{\prime}\right)<2^{-(m+n)}$. Moreover, we may suppose that $k(m+1, n)$ $\geqq k(m, n)$ for all $m, n$. Now let $b_{m}=\bigwedge_{n=1}^{\infty}\left(a_{m} \wedge b_{n, k(m, n)}\right)$. Clearly $b_{m} \leqq a_{m}$ and $b_{m} \uparrow$, because of the choice of $k(m, n)$. The construction of $b_{m}$ also ensures that $(\forall m) b_{m}$ $\ll\left\{b_{n, k}\right\}$. Finally

$$
a_{m} \wedge b_{m}^{\prime}=\bigvee_{n=1}^{\infty}\left(a_{m} \wedge b_{n, k(m, n)}^{\prime}\right)
$$

so that $\mu\left(a_{m} \wedge b_{m}^{\prime}\right)<2^{-m}$, and, $\mu$ being strictly positive on $\mathscr{B}$, it follows from $a_{m} \uparrow 1$ that $b_{m} \uparrow 1$. Thus the element $1 \in \mathscr{B}$ has the Egoroff property and, in view of Theorem 2.1(b), the proof is complete.

Together these theorems show that there is a good supply of Egoroff Boolean algebras and Riesz spaces, and incidentally include a proof of the classical Egoroff theorem which makes clear the role of the "Egoroff property" in this result. Taken with Theorem 1.1, these theorems yield Lorentz' observation that we have $\rho_{M}=\rho_{L}$ for every monotone seminorm $\rho$ on a Banach function space over a $\sigma$ finite measure space; at the same time we see that the weaker condition that the measure algebra $\mathscr{B}$ be Egoroff is sufficient for this result. Moreover, this weaker condition is necessary since for Archimedean Riesz spaces the notions of almostEgoroff and Egoroff coincide (see Theorem 4.5).

Concerning the relationship between the Egoroff property and $\sigma$-finiteness for a measure algebra $\mathscr{B}$ it should be remarked that under favorable circumstances the two are equivalent. More precisely, if $\mathscr{B}$ is Egoroff and complete (i.e., the measure space is "localizable") and if $\mu$ is locally finite (i.e., if $0 \neq b \in \mathscr{B}$, then $b$ majorizes a nontrivial element of finite measure), then $(\mathscr{B}, \mu)$ is $\sigma$-finite, at least if we assume the continuum hypothesis. Note that both of the additional conditions are, in fact, necessary if $(\mathscr{B}, \mu)$ is to be $\sigma$-finite. This result is due to Luxemburg (cf. [8, Theorems 4.6 and 2.1]). For the sake of completeness we include a sketch of a proof. Using Zorn's lemma, and the local finiteness of $\mu$, we easily construct a maximal family $\mathscr{D}$ of disjoint nontrivial elements of $\mathscr{B}$ of finite measure. It must be that $\sup \mathscr{D}=1$ so that if $\mathscr{D}$ is countable then, indeed, $(\mathscr{B}, \mu)$ is $\sigma$-finite. If, however, $\mathscr{D}$ is uncountable, then, assuming the continuum hypothesis, we must have a one-one mapping $\phi$ from $N^{N}$ (see Example 4.2 below) into $\mathscr{D}$. Let $b=\sup \left(\phi\left(N^{N}\right)\right)$ and $b_{n, k}=\sup \left(\phi\left(\left\{x: x \in N^{N}\right.\right.\right.$ and $\left.\left.\left.x(n) \leqq k\right\}\right)\right)$; using the argument of Example 4.2, we see that the double sequence $\left\{b_{n, k}\right\}$ violates the Egoroff property of the element $b$.

4. Relationships among the Egoroff conditions; examples. It is clear that if an element of a Boolean algebra has the Egoroff property, then it has also the weak 
Egoroff property; similarly the elements of a Riesz space which have the Egoroff property are included among those having the almost-Egoroff property, and all of these, in turn, certainly have the weak Egoroff property. The examples below, however, show that no two of these notions coincide.

EXAMPLE 4.1. $C[0,1]$, the set of continuous, real-valued functions on the interval $[0,1]$, becomes a Riesz space under the ordinary pointwise linear operations and order. It is easy to show that no positive element in this space has the weak Egoroff property. To see this, consider a function $u>0$. Let $\left\{r_{n}\right\}$ be an enumeration of the rationals in $[0,1]$, and, for each $n$, let $u_{n, k}$ be a sequence of functions in $C[0,1]$ such that, for each $x, 0 \leqq u_{n, k}(x) \uparrow_{k} u(x)$, unless $x \in\left\{r_{1}, r_{2}, \ldots, r_{n}\right\}$ in which case $u_{n, k}(x)=0$. Clearly $u_{n, k} \uparrow_{k} u$ in $L=C[0,1]$, while if $0 \leqq g \ll\left\{u_{n, k}\right\}$, then $g\left(r_{n}\right)=0$ for all $n$, so that, since $g$ is continuous, $g \equiv 0$.

EXAmple 4.2. Consider the space $R^{X}$ of all real functions over the set $X=N^{N}$ (all functions from $N=\{1,2, \ldots\}$ into $N$ ), with the natural pointwise linear operations and order. Define the following subsets of $X: X_{n, k}=\left\{x: x \in N^{N}\right.$ and $\left.x(n) \leqq k\right\}$. For each $n$, we have $X_{n, k} \uparrow_{k} X$ (in $R^{X}$ ); nevertheless, this double sequence has the rather surprising property that for no choice of $k(n)$ do we have $\sup X_{n, k(n)}=X$. In fact, we can construct $x \in X$ such that $(\forall n) x \notin X_{n, k(n)}$ simply by setting $x(n)$ $=k(n)+1$ for all $n \in N$. We can now see that the unit function in $R^{X}$ has not the Egoroff property, nor even the almost-Egoroff property. Suppose we have $(\forall m) 0$ $\leqq u_{m} \ll\left\{X_{n, k}\right\}$; then in particular we can choose $k(m)$, for each $m$, such that $u_{m} \leqq X_{m, k(m)}$. Since there exists $x \in X$ such that $(\forall m) X_{m, k(m)}(x)=0$, so that $\left[\sup u_{m}\right](x)$ $=0$ while $X(x)=1$, it is clear that $X$ cannot have the almost-Egoroff property. On the other hand, it is clear that the whole space $L$ is weak Egoroff. This discussion shows equally well that the power set $p\left(N^{N}\right)$ furnishes an example of a Boolean algebra which is weak Egoroff but not Egoroff.

EXAMPLE 4.3. For this example it is convenient to have available an nonArchimedean extension ${ }^{*} R$ of the real numbers $R$, i.e. we assume ${ }^{*} R$ to be a totally ordered field containing $R$ as a subfield and containing elements $h$, called infinitesimals, such that $h \neq 0$, but $|h|<r$ for every $r>0, r \in R$. A general construction for such $* R$ is discussed in [9]; however, all we really need here is a totally ordered non-Archimedean linear space over $R$.

Let $L$ consist of the functions $f$ on the set $X=N^{N}$ (see Example 4.2) with values in $* R$, and having the following form: $f(x)=r(f)+h_{f}(x)$, where $r(f) \in R$ and, for each $x \in X, h_{f}(x)$ is infinitesimal or zero. Upon the introduction of the pointwise linear operations and the pointwise ordering induced by the order in $* R, L$ becomes a Riesz space. Note that if $r(f)<r(g)$ then $f<g$, regardless of the values of the functions $h_{f}$ and $h_{g}$. Now the unit function $u$ in $L\left(r(u)=1, h_{u} \equiv 0\right)$ has the almostEgoroff property. To see this, suppose that $(\forall n) 0 \leqq u_{n, k} \uparrow_{k} u$; certainly $r\left(u_{n, k}\right) \uparrow_{k} r(u)$ $=1$, so that, given $\varepsilon>0$, we have some $k(n)$, for each $n$, such that $r\left(u_{n, k(n)}\right)>1-\varepsilon$ $=r((1-\varepsilon) u)$; thus, for any $\varepsilon>0,(1-\varepsilon) u \ll\left\{u_{n, k}\right\}$.

Consider now the element $h X$ in $L$, where $h$ is a fixed positive infinitesimal. 
Using the notation of Example 4.2 we have $h X_{n, k} \uparrow_{k} h X$ (for each $n$ ), and, arguing just as we did in the discussion of Example 4.2, we see that $h X$ has not the almostEgoroff property with respect to this double sequence. As $0 \leqq h X \leqq u$, we have shown that the almost-Egoroff property is not an ideal property; what is more important, we see that $u$ has not the Egoroff property, for in that case $h X$ would have that property as well, in view of Theorem 2.1(a). Thus we have exhibited an element which has the almost-Egoroff property but not the Egoroff property.

EXAMPLE 4.4. We can demonstrate the existence of a Boolean algebra $\mathscr{B}$ not having the weak Egoroff property, at least if we assume the continuum hypothesis. Consider the algebra $p(X)$ of all subsets of a set $X$ having cardinality $c$ (e.g., $X=[0,1])$. It was proved by Banach and Kuratowski (see [1]), as the basic tool in their demonstration of the nonmeasurability of the cardinal $c$, that, if the continuum hypothesis holds, there exist subsets $X_{n, k}$ of $X$ such that (i) $(\forall n) X_{n, k} \uparrow_{k} X$, and (ii) for any choice of $k(n), \bigcap_{n=1}^{\infty} X_{n, k(n)}$ is countable. This result is also discussed (as Proposition $C_{11}$ ) in Sierpinski's well-known book [11]. If we now form the quotient algebra $p(X) / C$, where $C$ is the ideal in $p(X)$ consisting of those subsets which are at most countable, we have our example $\mathscr{B}$. Indeed, if $h$ is the canonical map of $p(X)$ onto $\mathscr{B}$, it is evident that $(\forall n) h\left(X_{n, k}\right) \uparrow_{k} 1 \in \mathscr{B}$, while, for any choice of $k(n), \bigwedge_{n=1}^{\infty} h\left(X_{n, k(n)}\right)=0$. Thus 1 has not the weak Egoroff property and it is an easy consequence that no nonzero element of $\mathscr{B}$ has that property.

Thus we cannot hope to show that every Boolean algebra is weak Egoroff, since Gödel has shown (see [4]) that the continuum hypothesis is consistent with the other axioms of set theory (if those axioms are consistent). The importance for this example of the Banach-Kuratowski construction was pointed out by Professor Luxemburg.

We have now completed our list of examples designed to separate the various Egoroff conditions in Riesz spaces and Boolean algebras. It is important to note that the non-Archimedean character of the example (Example 4.3) of an element in a Riesz space having the almost-Egoroff property without the Egoroff property is essential, as the following result shows.

TheOREM 4.5. If $u \in L^{+}$is such that inf $\{(1 / n) u: n=1,2,3, \ldots\}=0$ (in particular, if the Riesz space $L$ is Archimedean), then $u$ has the Egoroff property if and only if $u$ has the almost-Egoroff property.

Proof. We have already noted that one implication always holds. For the other, suppose that $u$ has the almost-Egoroff property, and that $(\forall n) 0 \leqq u_{n, k} \uparrow_{k} u$. Choose a sequence of real numbers $\varepsilon_{p}$ and elements $u_{m}^{p}$ such that $\varepsilon_{p} \uparrow 1,0 \leqq u_{m}^{p} \uparrow_{m} \varepsilon_{p} u$, and $u_{m}^{p} \ll\left\{u_{n, k}\right\}$ (for all $m, p$ ). Now let $u_{m}=u_{m}^{1} \vee \cdots \vee u_{m}^{m}$. Clearly $0 \leqq u_{m} \uparrow$ and $u_{m} \leqq u$. Moreover, if $v$ is such that $(\forall m) u_{m} \leqq v$, then $(\forall m) u_{m}^{p} \leqq v$, so that $v \geqq \varepsilon_{p} u$; but sup $\varepsilon_{p} u$ $=u$, since $\varepsilon_{p} \uparrow 1$ and we have assumed that $u$ is an "Archimedean element". Hence $u_{m} \uparrow u$. Since $u_{m}^{p} \ll\left\{u_{n, k}\right\}$ for $p=1,2, \ldots, m$, it is clear that $u_{m} \ll\left\{u_{n, k}\right\}$ also. Thus $u$ has the Egoroff property. 
5. Central theorems. We now demonstrate the connection between the almostEgoroff property and the relation $\rho_{M}=\rho_{L}$ for monotone seminorms $\rho$ on a Riesz space $L$; included is the proof of Theorem 1.1. The existence of $\rho_{M}$ for every monotone seminorm $\rho$ is easily established; in fact, we can construct $\rho_{M}(u)$ as $\sup \left\{\rho_{1}(u): \rho_{1}\right.$ is a $\sigma$-Fatou monotone seminorm dominated by $\left.\rho\right\}$, simply verifying that this $\rho_{M}$ is again a $\sigma$-Fatou monotone seminorm. It is also easy to check that the "Lorentz seminorm" $\rho_{L}$ is indeed a monotone seminorm for each $\rho$; moreover, $\rho$ is $\sigma$-Fatou precisely when $\rho_{L}=\rho$. Thus, since $\rho \geqq \rho_{M}$, we have $\rho_{L} \geqq \rho_{M L}=\rho_{M}$, so that $\rho_{L}=\rho_{M}$ if and only if $\rho_{L}$ is $\sigma$-Fatou, i.e., $\rho_{L L}=\rho_{L}$. This accounts for the form of the following theorems.

THEOREM 5.1. If $f$ is an element of $L^{+}$and $f$ has the almost Egoroff property, then for every monotone seminorm $\rho$ we have $\rho_{L L}(f)=\rho_{L}(f)$.

Proof. It is clear that $\rho_{L L}(f) \leqq \rho_{L}(f)$. On the other hand, suppose $\lambda>\rho_{L L}(f)$; in this case there must exist $0 \leqq f_{n} \uparrow_{n} f$ and, for each $n, 0 \leqq f_{n, k} \uparrow_{k} f_{n}$ such that $\rho\left(f_{n, k}\right)$ $<\lambda$ for all $n, k$. Now if we let $g_{n, k}=f_{n, k}+\left(f-f_{n}\right)$, we have $g_{n, k} \uparrow_{k} f$, for each $n$. Since $f$ has the almost-Egoroff property there exists, for each $\varepsilon(0<\varepsilon \leqq 1)$, a sequence $f_{m}^{\varepsilon}$ such that $0 \leqq f_{m}^{\varepsilon} \uparrow_{m}(1-\varepsilon) f$ and $f_{m}^{\varepsilon} \ll\left\{g_{n, k}\right\}$ for all $m$. Now if we set $h_{m}^{\varepsilon}=$ $\left(f_{m}^{\varepsilon}+f_{m}-f\right)^{+}$, we have $h_{m}^{\varepsilon} \uparrow_{m}(1-\varepsilon) f$. Moreover, for each $m$ there is some $k(m)$ such that $f_{m}^{\varepsilon} \leqq g_{m, k(m)}$ and $h_{m}^{\varepsilon} \leqq g_{m, k(m)}+f_{m}-f=f_{m, k(m)}$. We then have $\rho\left(h_{m}^{\varepsilon}\right) \leqq$ $\rho\left(f_{m, k(m)}\right)<\lambda$, so that $\rho_{L}((1-\varepsilon) f) \leqq \lambda$, i.e. $\rho_{L}(f) \leqq(1-\varepsilon)^{-1} \lambda$. Since this is true for every $\varepsilon>0$, we have $\rho_{L}(f) \leqq \lambda$; hence $\rho_{L}(f) \leqq \rho_{L L}(f)$.

We now prove a strong converse to Theorem 5.1 ; the full strength of this result will be useful in our subsequent discussion.

THEOREM 5.2. Let $f$ be an element of $L^{+}$, where $L$ is a Riesz space; if $\rho_{L L}(f)=\rho_{L}(f)$ for every monotone seminorm $\rho$ on $L$ such that $\rho(f)<\infty$, then $f$ has the almostEgoroff property.

Proof. Suppose that $0 \leqq f_{n, k} \uparrow_{k} f$, for each $n$. Let

$$
h_{n, k}=f_{1, k} \wedge f_{2, k} \wedge \cdots \wedge f_{n, k}
$$

then $h_{n, k} \uparrow_{k} f$, for each $n$, and $[n \leqq m] \Rightarrow\left[h_{n, k} \geqq h_{m, k}\right]$. We shall show that, given $\varepsilon$ $(1 \geqq \varepsilon>0)$, there exists a sequence $f_{m}^{\varepsilon}$ such that $0 \leqq f_{m}^{\varepsilon} \uparrow_{m}(1-\varepsilon) f$ and $f_{m}^{\varepsilon} \ll\left\{h_{n, k}\right\}$, for each $m$; since $h_{n, k} \leqq f_{n, k}$ we also have $f_{m}^{\varepsilon} \ll\left\{f_{n, k}\right\}$.

(a) First we show that, given any $\varepsilon_{1}>0$, there exists a sequence $g_{n}$ such that $0 \leqq g_{n} \uparrow f$ and $\left[(\exists n) M f \leqq g_{n}\right] \Rightarrow\left[M \leqq \varepsilon_{1}\right]$. For each $n$, let $\beta_{n}=\sup \left\{\beta:(\exists k) \beta f \leqq h_{n, k}\right\}$. Clearly, for all $n, \beta_{n} \geqq 0$; on the other hand, if $\beta_{n} \geqq 1$ for all $n$, then we can construct $f_{m}^{\varepsilon}$ immediately by setting $f_{m}^{\varepsilon}=(1-\varepsilon) f$, since $\left[(\forall n)(1-\varepsilon)<\beta_{n}\right] \Rightarrow(1-\varepsilon) f \ll\left\{h_{n, k}\right\}$. We can assume, then, that there exists $p$ such that $0 \leqq \beta_{p}<1$. Consider, for $\beta<\beta_{p}$, the sequence $h_{k}=(1-\beta)^{-1}\left(h_{p, k}-\beta f\right)$; clearly $h_{k} \uparrow_{k} f$ and the terms of the sequence are nonnegative after a finite number, since $\beta<\beta_{p}$. Denote the nonnegative tail of the 
sequence $\left\{h_{k}\right\}$ by $g_{1}, g_{2}, \ldots$ If there exists $k$ such that $M f \leqq g_{k}$, then there is a corresponding $k^{\prime}$ such that $M f \leqq(1-\beta)^{-1}\left(h_{p, k^{\prime}}-\beta f\right)$ and it easily follows that $M \leqq$ $(1-\beta)^{-1}\left(\beta_{p}-\beta\right)$. Thus, by taking $\beta$ sufficiently close to $\beta_{p}$, we can ensure that $\left[(\exists n) M f \leqq g_{n}\right] \Rightarrow\left[M \leqq \varepsilon_{1}\right]$.

(b) Next, given $0<\varepsilon_{2}<1$, we set $g_{n, k}=\left(h_{n, k} \wedge g_{n}\right) \vee \varepsilon_{2} f$. Then, for each $n$,

$$
g_{n, k} \uparrow_{k}\left(\left(f \wedge g_{n}\right) \vee \varepsilon_{2} f\right)=g_{n} \vee \varepsilon_{2} f,
$$

and $\left(g_{n} \vee \varepsilon_{2} f\right) \uparrow_{n}\left(f \vee \varepsilon_{2} f\right)=f$. Now, for $x \in L^{+}$, set $\rho(x)=\inf \left\{\sum_{n} \alpha_{n}: \alpha_{n} \geqq 0\right.$ and $\sum_{n} \alpha_{n} g_{n, k(n)} \geqq x$ for some $\left.k(n)\right\}$; the sums involved are understood to be finite, i.e., all but a finite number of the $\alpha_{n}$ are zero; set $\rho(x)=+\infty$ if there is no such finite sum covering $x . \rho$ is a monotone seminorm on $L$. That $\rho$ is monotone and positive homogeneous is clear. $\rho$ is also sublinear, for if $\lambda>\rho(x)+\rho(y)$, then there exist $\alpha_{n}, k(n), \gamma_{n}, j(n)$ such that $\lambda>\sum_{n} \alpha_{n}+\sum_{n} \gamma_{n}$ and $\sum_{n} \alpha_{n} g_{n, k(n)} \geqq x, \sum_{n} \gamma_{n} g_{n, j(n)} \geqq y$; thus

$$
\sum_{n}\left(\alpha_{n}+\gamma_{n}\right) g_{n, \max (k(n), y(n))} \geqq x+y
$$

so that $\rho(x+y) \leqq \sum_{n}\left(\alpha_{n}+\gamma_{n}\right)<\lambda$. Moreover, $\rho(f)<\infty$; in fact, $g_{1,1} \geqq \varepsilon_{2} f$, so that $\rho(f) \leqq\left(\varepsilon_{2}\right)^{-1}$.

Now $\rho\left(g_{n, k}\right) \leqq 1$ so that, since $g_{n, k} \uparrow_{k}\left(g_{n} \vee \varepsilon_{2} f\right)$, we have $\rho_{L}\left(g_{n} \vee \varepsilon_{2} f\right) \leqq 1$, for all $n$. But $\left(g_{n} \vee \varepsilon_{2} f\right) \uparrow_{n} f$, so that $\rho_{L L}(f) \leqq 1$. Hence, by our assumption, $\rho_{L}(f) \leqq 1$. This means that, for any $\varepsilon_{3}>0$, there exists a sequence $f_{m}$ such that $0 \leqq f_{m} \uparrow_{m} f$ and, for each $m, \rho\left(f_{m}\right)<1+\varepsilon_{3}$.

(c) If we now set $f_{m}^{\varepsilon}=\left(f_{m}-\varepsilon f\right)^{+}$, we have $0 \leqq f_{m}^{\varepsilon} \uparrow_{m}(1-\varepsilon) f$. The remainder of the proof consists in showing that, by appropriate choices of $\varepsilon_{1}, \varepsilon_{2}, \varepsilon_{3}$, we can ensure that $f_{m}^{\varepsilon} \ll\left\{h_{n, k}\right\}$.

Let us find, then, conditions on $\varepsilon_{1}, \varepsilon_{2}, \varepsilon_{3}$ which imply that, for a particular $m_{1}$, $n_{1}$, there exists some $k$ such that $f_{m_{1}}^{\varepsilon} \leqq h_{n_{1}, k}$; we shall find conditions independent of $m_{1}, n_{1}$, thereby establishing our result.

Now $\rho\left(f_{m}\right)<1+\varepsilon_{3}$ so that there exist $\alpha_{n}^{m} \geqq 0, k^{m}(n)$ such that $\sum_{n} \alpha_{n}^{m}<1+\varepsilon_{3}$ and $\sum_{n} \alpha_{n}^{m} g_{n, k^{m}(n)} \geqq f_{m}$. If we now let $\gamma=\inf \left\{\left(\sum_{n<n_{1}} \alpha_{n}^{m}\right): m \geqq m_{1}\right\}$, then, for $m \geqq m_{1}$,

$$
\sum_{n \geq n_{1}} \alpha_{n}^{m}=\sum_{n} \alpha_{n}^{m}-\sum_{n<n_{1}} \alpha_{n}^{m}<1+\varepsilon_{3}-\gamma .
$$

Thus $\sum_{n \geqq n_{1}} \alpha_{n}^{m} g_{n, k^{m}(n)} \leqq\left(1+\varepsilon_{3}-\gamma\right) f$ so that

$$
\begin{aligned}
f_{m}-\left(1+\varepsilon_{3}-\gamma\right) f & \leqq f_{m}-\sum_{n \geqq n_{1}} \alpha_{n}^{m} g_{n, k^{m}(n)} \\
& \leqq \sum_{n \leqq n_{1}} \alpha_{n}^{m} g_{n, k^{m}(n)} \leqq\left(1+\varepsilon_{3}\right)\left(g_{n_{1}} \vee \varepsilon_{2} f\right) \leqq\left(1+\varepsilon_{3}\right)\left(g_{n_{1}}+\varepsilon_{2} f\right) .
\end{aligned}
$$

But $f=\sup \left\{f_{m}: m \geqq m_{1}\right\}$ so that we have

i.e.

$$
f-\left(1+\varepsilon_{3}-\gamma\right) f \leqq\left(1+\varepsilon_{3}\right)\left(g_{n_{1}}+\varepsilon_{2} f\right),
$$

$$
\left(\left(\gamma-\varepsilon_{3}\right)\left(1+\varepsilon_{3}\right)^{-1}-\varepsilon_{2}\right) f \leqq g_{n_{1}} .
$$


Hence, by the consiruction of part (a), $\left(\gamma-\varepsilon_{3}\right)\left(1+\varepsilon_{3}\right)^{-1}-\varepsilon_{2} \leqq \varepsilon_{1}$. Clearly, then, if we let $\varepsilon_{4}=\left(1+\varepsilon_{3}\right)\left(\varepsilon_{1}+\varepsilon_{2}\right)+2 \varepsilon_{3}$, there must exist $m \geqq m_{1}$ such that $\sum_{n<n_{1}} \alpha_{n}^{m}<\varepsilon_{4}$. Now, for such an $m \geqq m_{1}$, we have

$$
\begin{aligned}
f_{m} & \leqq \varepsilon_{4} f+\sum_{n \geqq n_{1}} \alpha_{n}^{m} g_{n, k^{m}(n)} \\
& \leqq \varepsilon_{4} f+\sum_{n \geqq n_{1}} \alpha_{n}^{m}\left(h_{n, k^{m}(n)}+\varepsilon_{2} f\right) .
\end{aligned}
$$

If we let $k=\max \left\{k^{m}(n): \alpha_{n}^{m} \neq 0\right\}$ and recall that, for $n \geqq n_{1}, h_{n, k} \leqq h_{n_{1}, k}$, we see that

$$
f_{m} \leqq \varepsilon_{4} f+\left(\sum_{n \geqq n_{1}} \alpha_{n}^{m}\right)\left(h_{n_{1}, k}+\varepsilon_{2} f\right) .
$$

Now $m \geqq m_{1}$ so that we have

$$
\begin{aligned}
f_{m_{1}} & \leqq f_{m} \leqq \varepsilon_{4} f+\left(1+\varepsilon_{3}\right)\left(h_{n_{1}, k}+\varepsilon_{2} f\right) \\
& \leqq\left(\varepsilon_{4}+\varepsilon_{3}+\left(1+\varepsilon_{3}\right) \varepsilon_{2}\right) f+h_{n_{1}, k} \leqq \varepsilon f+h_{n_{1}, k}
\end{aligned}
$$

provided we choose $\varepsilon_{1}, \varepsilon_{2}, \varepsilon_{3}$ such that $\left(\left(1+\varepsilon_{3}\right)\left(\varepsilon_{1}+2 \varepsilon_{2}\right)+3 \varepsilon_{3}\right) \leqq \varepsilon$, which we clearly can do. In this case, $f_{m_{1}}^{\varepsilon}=\left(f_{m_{1}}-\varepsilon f\right)^{+} \leqq h_{n_{1}, k}$, as required.

Theorem 1.1 follows directly from Theorems 5.1 and 5.2; note that, in view of Theorem 4.5, we can replace the almost-Egoroff condition in Theorem 1.1 by the simple Egoroff condition, provided we consider only Archimedean Riesz spaces.

6. Comments. (a) We have seen that on Riesz spaces which are not almostEgoroff there must exist monotone seminorms $\rho$ which are "singular" in the sense that $\rho_{L L} \neq \rho_{L}$; in certain cases such $\rho$ may actually be "Riesz norms", i.e. we may have $(\forall u \neq 0)(0<\rho(u)<\infty)$. For example, in the Riesz space $C[0,1]$, the unit function $u$ certainly has not the almost-Egoroff property (see Example 4.1). Hence, there exists a monotone seminorm $\rho$, with $\rho(u)$ finite, such that $\rho_{L L}(u) \neq \rho_{L}(u)$ Theorem 5.2). Since the ideal generated by $u$ is all of $C[0,1], \rho$ is everywhere finite and to obtain our singular Riesz norm on $C[0,1]$ we simply add the uniform norm to $\rho$.

(b) In his paper [5] of $1958 \mathrm{~S}$. Koshi proved that, under certain conditions on a Boolean algebra $\mathscr{B}$, every finitely additive measure on $\mathscr{B}$ is countably additive on a suitable super order dense ideal (a subset $A$ of a partially ordered system $X$ is said to be super order dense if every element in $X$ is the supremum of an increasing sequence taken from $A$ ). Luxemburg subsequently showed that the Egoroff condition on $\mathscr{B}$ was sufficient for this result (cf. [8], Theorem 5.1), and a number of similar theorems dealing with linear functionals and seminorms on Riesz spaces have appeared (see [6, Note VI, Corollary 20.7], [7, Note XIV, Theorem 44.2], and [7, Note XVI, Theorem 64.9]). The Egoroff condition is assumed in these theorems, but often it can be replaced by the almost-Egoroff condition. These results converge on the following question: if $L$ is almost-Egoroff, does it follow 
that $\rho=\rho_{L}$ on some order dense ideal $I_{\rho}$, for every monotone seminorm $\rho$ ? While this question apparently remains unanswered, we can see that such a result would be best possible. This follows from Theorem 5.2 since if $\rho_{L}=\rho$ on a super dense ideal, it is easy to show that $\rho_{L L}=\rho_{L}$ (throughout the space); hence if, for every $\rho$, $\rho_{L}=\rho$ on a super order dense ideal $I_{\rho}$, our theorem ensures that the space must be almost-Egoroff.

(c) For any monotone function $\rho: \mathscr{B} \rightarrow[0, \infty]$ on a Boolean algebra $\mathscr{B}$ we can define $\rho_{L}$ just as in the Riesz space case. We then have the following analogue of Theorem 1.1: $\mathscr{B}$ is Egoroff if, and only if, $\rho_{L L}=\rho_{L}$ for every (finite-valued) outer measure $\rho$ on $\mathscr{B}$. The argument proceeds along the lines of the proofs of Theorems 5.1 and 5.2, but in the Boolean algebra case the techniques are needed only in a rudimentary form.

\section{REFERENCES}

1. S. Banach and C. Kuratowski, Sur une généralisation du problème de mesure, Fund. Math. 14 (1929), 127-131.

2. N. Bourbaki, Intégration, Actualités Sci. Indust. No. 1175, Hermann, Paris, 1952.

3. D. T. Egoroff, Sur les suites des fonctions mesurables, C. R. Acad. Sci. Paris 152 (1911), 244-246.

4. K. Gödel, The consistency of the continuum hypothesis, Ann. of Math. Studies No. 3, Princeton Univ. Press, Princeton, N. J., 1940.

5. S. Koshi, On semicontinuity of functionals. I, Proc. Japan Acad. 34 (1958), 513-517.

6. W. A. J. Luxemburg and A. C. Zaanen, Notes on Banach function spaces, Proc. Acad. Sci. Amsterdam; Note I, A66 (1963), 135-147; Note II, A66, 148-153; Note III, A66, 239-250; Note IV, A66, 251-263; Note V, A66, 496-504; Note VI, A66, 655-668; Note VII, A66, 669-681; Note VIII, A67 (1964), 104-119; Note IX, A67, 360-376; Note X, A67, 493-506; Note XI, A67, 507-518; Note XII, A67, 519-529; Note XIII, A67, 530-543.

7. W. A. J. Luxemburg, Notes on Banach function spaces, Proc. Acad. Sci. Amsterdam: Note XIV, A68 (1965), 229-248; Note XV, A68, 415-446; Note XVI, A68, 646-667.

8. - On finitely additive measures in Boolean algebras, J. Reine Angew. Math. 213 (1964), 165-173.

9. - Non-standard analysis, 2nd ed., California Inst. of Tech., Pasadena, Calif., 1964.

10. H. Nakano, Modulared semiordered linear spaces, Maruzen, Tokyo, 1950.

11. W. Sierpiński, Hypothèse du continu, Monogr. Mat., Warsaw, 1934.

\section{California Institute of Technology, Pasadena, California}

